\title{
Influência do selamento dentinário imediato com sistema adesivo universal na resistência de união
}

\author{
Influence of immediate dentin sealing on dentin bond strength with universal \\ adhesive system
}

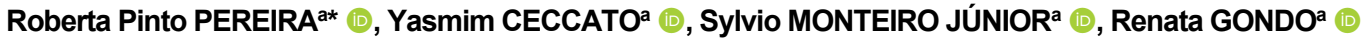 \\ aUFSC - Universidade Federal de Santa Catarina, Departamento de Odontologia, Florianópolis, SC, Brasil
}

\begin{abstract}
Como citar: Pereira RP, Ceccato Y, Monteiro Júnior S, Gondo R. Influência do selamento dentinário imediato com sistema adesivo universal na resistência de união. Rev Odontol UNESP. 2020;49:e20200071. https://doi.org/10.1590/18072577.07120
\end{abstract}

\begin{abstract}
Resumo
Introdução: 0 selamento dentinário imediato consiste na aplicação imediata de um sistema adesivo sobre exposições dentinárias após o preparo dental e previamente aos procedimentos de moldagem. Objetivo: 0 objetivo do estudo foi avaliar a influência do selamento dentinário imediato (SDI) na resistência adesiva do sistema adesivo universal (Single Bond Universal, 3M ESPE) em dentina, na forma de aplicação autocondicionante. Material e método: 30 incisivos bovinos foram divididos em 3 grupos $(n=10)$ : grupo $S$ (Sem Selamento Dentinário Imediato), grupo SDI (Selamento Dentinário Imediato) e grupo SDIF (Selamento Dentinário Imediato + Resina Flow). A superfície vestibular foi desgastada com pontas diamantadas até a exposição superficial de dentina. A simulação da lama dentinária foi realizada com lixa d'água por 30s e a dentina tratada de acordo com o respectivo grupo. Para simulação da restauração provisória, foram cimentados blocos de resina bisacrílica com cimento de óxido de zinco. Após 7 dias, foi realizada a cimentação definitiva de blocos de resina com cimento resinoso dual (RelyX ARC, 3M ESPE). Os espécimes foram seccionados para obtenção de filetes de $1 \mathrm{~mm}^{2}$ e submetidos ao teste de microtração, em uma máquina universal de ensaios (Instron). Os resultados foram tabulados e submetidos a Análise de Variância (ANOVA) e teste de Tukey a $5 \%$ de significância. Resultado: Observaram-se maiores valores de resistência de união para o grupo SDIF (23,53 MPa), diferindo estatisticamente do Grupo S (17,65 MPa) (p=0,008). 0 grupo SDI apresentou valor médio de resistência de união $(19,25 \mathrm{MPa})$, não diferindo estatisticamente dos grupos $\mathrm{S}(\mathrm{p}=0,68)$ e SDIF $(p=0,07)$. Conclusão: Recomenda-se a técnica de selamento dentinário imediato seguido da uma camada de resina flow, em exposições dentinárias ocasionadas pelo preparo dental para restaurações indiretas.
\end{abstract}

Descritores: Hibridização dentinária; adesivos; cimentação.

\begin{abstract}
Introduction: Imediate dentin sealing (IDS) is a procedure where a dentin adhesive is applied on the exposed dentin surfaces after its preparation to indirect restorations and before impression procedures. Objective: The objective of the study was to evaluate the effectiveness of the immediate dentin sealing technique (SDI) in adhesive resistance, using the Universal adhesive system (Single Bond Universal, 3M ESPE). Material and method: 30 bovine incisors were selected and divided into 3 groups $(\mathrm{n}=10)$ : Groups S (Without Immediate Dentin Seal), SDI (Immediate Dentin Seal with Adhesive) and SDIF (Immediate Dentin Seal with Adhesive + Flow Resin). The vestibular surface was worn with diamond-tipped conical tips until superficial dentin exposure. The smear layer simulation was carried out with \# 600 granulation sandpaper for 30 s and the dentin was treated according to the respective group. To simulate the temporary restoration, blocks of bisacrylic resin were cemented with zinc oxide cement. After 7 days, the final cementation of resin blocks with dual resin cement (RelyX ARC, 3M ESPE) was carried out. The specimens were sectioned to obtain $1 \mathrm{~mm} 2$ fillets and subjected to the microtensile test. Result: The ANOVA test showed a statistical difference between the groups evaluated. Higher values of bond strength were observed for the SDIF group (23.53 MPa), differing statistically from Group S $(17,65 \mathrm{MPa})(\mathrm{p}=0.008)$. The SDI group showed an average bond strength $(19.25 \mathrm{MPa})$, which did not differ statistically from groups $S(p=0.68)$ and $\operatorname{SDIF}(p=0.07)$. Conclusion: Based on the results, it is recommended the immediate dental sealing technique with adhesive + flow resin in dental exposures caused in the dental preparation for indirect restorations.
\end{abstract}

Descriptors: Dentin hybridization; adhesives; cementation. 


\section{INTRODUÇÃO}

O sucesso clínico de procedimentos restauradores indiretos está condicionado a vários fatores, entre eles, à força de adesão entre os tecidos dentais e a restauração, através do uso de sistemas adesivos ${ }^{1}$. Nessa perspectiva, foi proposto que as exposições dentinárias promovidas pelo preparo cavitário fossem seladas imediatamente após a conclusão do desgaste, através da utilização de um agente adesivo, o qual seria aplicado e fotoativado previamente aos procedimentos de moldagem ${ }^{1}$.

De acordo com a literatura, a adoção desse método permite que a formação da camada híbrida ocorra sobre uma área de dentina recém-exposta, portanto, livre de contaminantes e compreendida como substrato ideal para a realização de procedimentos adesivos ${ }^{2-4}$. Ademais, quando se opta pelo tratamento com selamento dentinário, a resistência de adesão à dentina e a adaptação marginal em restaurações cimentadas é maior ${ }^{1,5,6}$, e a ocorrência de sensisibilidade pós-operatória diminui 7 .

A técnica original recomenda que o selamento dentinário imediato seja realizado com sistemas adesivos de 3 passos, que utilizam o condicionamento ácido prévio e a aplicação de uma camada de resina hidrófoba sobre o primer. Esta camada hidrófoba é importante para reduzir ou impedir a permeabilidade da dentina a curto e longo prazo $^{8}$. Entre as marcas comerciais, o adesivo Optibond FL (Kerr, CA, EUA) é considerado um padrão ouro para a realização da técnica, com resultados efetivos por sua alta carga e alta resistência mecânica, resultando em maiores valores de resistência de união ${ }^{4,9}$. Apesar do seu bom desempenho, esse sistema não é comercializado em todos os países, e em razão das taxas de importação e seu alto custo, sua indicação, muitas vezes, pode ser inviável.

Com o desenvolvimento de adesivos simplificados, torna-se interessante sua indicação para o selamento dentinário imediato. Os adesivos autocondicionantes apresentam excelente resistência de união à dentina, com uma técnica simplificada de aplicação e menos sensível. Embora não tenha a mesma quantidade de carga que os adesivos convencionais, seu uso pode ser melhorado com a aplicação de uma camada de resina de baixa viscosidade (flow) após a hibridização ${ }^{10,11}$, funcionando como um material elástico, com mais carga que os adesivos simplificados, capaz de prevenir a ruptura do adesivo no ato da moldagem e na remoção do material restaurador provisório ${ }^{10}$. Além disso, a resina flow pode proteger a camada híbrida do estresse de contração do cimento resinoso durante sua polimerização e aumentar o grau de conversão dos adesivos dentinários, aumentando a resistência de união da interface adesiva ${ }^{11}$.

Considerando o exposto, percebe-se a necessidade de se compreender melhor tanto a técnica de execução do selamento dentinário imediato, quanto a sua sensibilidade às diferentes possibilidades de sistemas adesivos. Dessa forma, o objetivo deste estudo foi avaliar a efetividade da técnica de selamento dentinário imediato (SDI) na resistência adesiva, mediante o uso do sistema adesivo universal.

\section{MATERIAL E MÉTODO}

Foram selecionados 30 incisivos bovinos hígidos extraídos. Como critério de seleção, dentes com trincas e/ou lesões de cárie foram excluídos. Após a limpeza, foi realizada a remoção da porção radicular e da borda incisal dos dentes com disco de carburundum (American Burrs, Palhoça, SC, Brasil) acoplado à peça reta. Após o corte da raiz, houve exposição da cavidade pulpar, a qual teve seu conteúdo removido com auxílio de uma cureta de dentina no17. Dessa forma, foram obtidos blocos de dentes bovinos de aproximadamente $13 \mathrm{~mm}$ de comprimento e $9 \mathrm{~mm}$ de diâmetro. Os blocos foram armazenados em água, em temperatura ambiente. 
Para preenchimento da câmara pulpar, a dentina foi condicionada com ácido fosfórico a 37\% (Power Etching; BM4, Palhoça, SC, Brasil) durante 15s, seguido de enxágue com água por 60s e secagem com papel absorvente. Foi feita a aplicação do sistema adesivo (Single Bond Universal Adesivo, 3M ESPE, St. Paul, MN, EUA) de acordo com as instruções do fabricante. Os incrementos de resina cor A1 (Vittra APS, FGM, Joinvile, SC, Brasil) foram inseridos e, sequencialmente, fotoativados (Bluephase C5, Ivoclar Vivadent, Schaan, Liechtenstein) por 20s. Essa manobra de preenchimento possibilitou a obtenção de corpos de prova de comprimento adequado para realização do teste de microtração.

Os espécimes foram aleatoriamente divididos em 3 grupos $(n=10)$, conforme descritos na Tabela 1, de acordo com a técnica de selamento dentinário imediato, utilizando o sistema adesivo Single Bond Universal (3M ESPE, St. Paul, MN, EUA).

Tabela 1. Divisão dos grupos

\begin{tabular}{cc}
\hline Grupos & Tratamento da superfície dentinária \\
\hline S & Sem selamento dentinário \\
SDI & Selamento dentinário imediato com adesivo universal \\
SDIF & Selamento dentinário imediato com adesivo universal e recobrimento com resina flow \\
\hline
\end{tabular}

Os espécimes foram posicionados e fixados com cera sobre a superfície de cilindros de PVC, com dimensões de $2,5 \mathrm{~cm}$ de diâmetro por $2 \mathrm{~cm}$ de altura, preenchidos com resina acrílica quimicamente ativada (Vipi Flash, Vipi Ind. e Com. de Produtos Odontológicos Ltda; Pirassununga, SP, Brasil), de modo que a superfície de dentina exposta ficasse posicionada horizontalmente. Assim, foi possível simular a cimentação e facilitar o manuseio do espécime.

Com o auxílio de uma ponta diamantada tronco cônica 4138 (KG Sorensen, São Paulo, SP, Brasil), em alta rotação e sob refrigeração intensa, a superfície vestibular dos dentes foi desgastada até a exposição da dentina. Na sequência, a superfície foi polida por 30 s com lixas d'água de granulação \#600 em uma politriz (Panambra Struers DP-10, Panambra, São Paulo, Brasil), a fim de regularizar a área e padronizar a lama dentinária. Cada dente foi preparado individualmente imediatamente antes do procedimento adesivo.

Após o preparo, cada espécime foi tratado de acordo com seu respectivo grupo:

\section{Grupo S - Sem Selamento Dentinário Imediato}

Este grupo não recebeu tratamento da superfície dentinária após o preparo dental.

\section{Grupo SDI - Selamento Dentinário Imediato}

Imediatamente após o preparo para exposição de dentina, foi realizado o condicionamento seletivo do esmalte com ácido fosfórico 37\% (Power Etching, BM4, Maringá, PR, Brasil) por 30s, seguido de enxágue com spray de ar/água durante $60 \mathrm{~s}$ e secagem. Com o auxílio de um microaplicador (KG Brush, KG Sorensen, São Paulo, SP, Brasil), o sistema adesivo Single Bond Universal (3M ESPE, St. Paul, MN, EUA) foi aplicado e esfregado cuidadosamente sobre a dentina por 20s, seguido por suave jato de ar por 5s. Uma segunda camada do sistema adesivo, com o mesmo protocolo, foi aplicada. Posteriormente, foi realizada a fotoativação (Bluephase C5, Ivoclar Vivadent, Schaan, Liechtenstein) por 20s, seguida de uma camada de gel hidrossolúvel (Power Block, BM4, Maringá, Brasil) e fotoativação por 20s. 


\section{Grupo SDIF - Selamento dentinário Imediato + Resina flow}

Neste grupo, em cada espécime foi realizado o mesmo protocolo de aplicação do sistema adesivo na dentina recém-preparada, conforme o grupo anterior SDI. Além disso, concluído o protocolo de aplicação do sistema adesivo Single Bond Universal (3M ESPE, St. Paul, MN, EUA), uma discreta camada de resina fluida (Filtek Z350 Flow, 3M ESPE, St. Paul, MN, EUA) foi aplicada com auxílio de um microaplicador (KG Brush, KG Sorensen, São Paulo, SP, Brasil). Completou-se a sequência fotoativando-a (Bluephase C5, Ivoclar Vivadent, Schaan, Liechtenstein) por 20s, aplicação do gel hidrossolúvel (Power Block, BM4, Maringá, Brasil), seguido de fotopolimerização por 20s.

\section{Restauração Provisória}

Com o objetivo de simular as restaurações provisórias, foram confeccionados 30 blocos de resina bisacrílica (Protemp 4, 3M ESPE, St. Paul, MN, EUA) a partir de um molde de silicone de adição (Express XT, 3M ESPE, St. Paul, MN, EUA) com 8mm de diâmetro e 3mm de espessura. Os blocos foram cimentados sobre os dentes, identificados de acordo com cada grupo, com cimento provisório Temp Bond NE (Kerr Corp, Orange, Califórnia, EUA) manipulado e espalhado homogeneamente. Concluída a cimentação, os corpos de prova foram armazenados em ambiente úmido e permaneceram nessa condição por 7 dias.

\section{Cimentação Definitiva}

Com o auxílio de uma sonda exploradora $\mathrm{n}^{\mathrm{o}}$ 5, foi realizada a remoção do bloco de resina bisacrílica. Os resíduos de cimento provisório que permaneceram na superfície dentária foram cuidadosamente removidos com auxílio de curetas de dentina $\mathrm{n}^{\mathrm{0}} \mathbf{5}$, seguido de profilaxia com escova robinson e água em baixa rotação.

Com o objetivo de simular as restaurações indiretas, foram confeccionados 30 blocos de resina composta cor A3 (Charisma Classic, Heraeus Kulzer, Hanau, Alemanha) a partir de um molde de silicone de adição ( $8 \mathrm{~mm} \times 3 \mathrm{~mm})$. A cada incremento acrescentado, e após a conclusão da confecção do bloco, foi realizada a fotoativação por 40s (Bluephase C5, Ivoclar Vivadent, Schaan, Liechtenstein).

Cada bloco de resina composta recebeu tratamento da sua superfície, imediatamente antes da cimentação definitiva, através do jateamento de óxido de alumínio com partículas de 100 microns (Bio-Art, São Carlos, SP, Brasil), a 2,5cm de distância e 80 bar de pressão por 10s. Posteriormente, foi realizado o enxágue abundante com água e secagem com jato de ar. A limpeza do bloco ocorreu por meio da aplicação do ácido fosfórico a 37\% (Power Etching, BM4, Maringá, PR, Brasil) por 30s na sua superfície de cimentação. Após, foi realizada lavagem abundante, secagem com jatos de ar e aplicação de silano (Silano, Maquira, Maringá, Paraná, Brasil) com o auxílio de um microaplicador (KG Brush, KG Sorensen, São Paulo, SP, Brasil) durante 60s. 0 preparo do bloco foi concluído com aplicação do sistema adesivo (Single Bond Universal, 3M ESPE, St. Paul, MN, EUA) através de um microaplicador (KG Brush, KG Sorensen, São Paulo, SP, Brasil).

Para o tratamento da superfície dental para cimentação, foi realizado jateamento da superfície com óxido de alumínio com partículas de 100 microns (Bio-Art, São Carlos, SP, Brasil), a 2,5 cm de distância e 80 bar de pressão por 10 segundos, seguido de enxágue abundante com água e secagem com jato de ar.

Para tratamento de esmalte e dentina do Grupo $\mathrm{S}$, foi realizado o condicionamento com ácido fosfórico 37\% (Power Etching, BM4, Maringá, PR, Brasil). 0 esmalte foi condicionado por 30s e a dentina por $15 \mathrm{~s}$, seguido de enxágue com spray de ar/água durante 60 s e secagem com discos de 
papel absorvente. Na sequência, foi realizada a aplicação do sistema adesivo Single Bond Universal (3M ESPE, St. Paul, MN, EUA), que foi esfregado cuidadosamente na dentina recémpreparada por 20s, seguido de suaves jatos de ar por $5 \mathrm{~s}$, nova camada de aplicação e evaporação com jatos de ar, e fotoativação (Bluephase C5, Ivoclar Vivadent, Schaan, Liechtenstein) por 20s. Completou-se a sequência fotoativando-o por mais 20s, após aplicação do gel hidrossolúvel (Power Block, BM4, Maringá, Brasil).

Para o tratamento dos tecidos dentais dos grupos SDI e SDIF, o mesmo protocolo descrito no grupo $\mathrm{S}$ foi realizado. Porém, a limpeza da superfície, o condicionamento e a aplicação do sistema adesivo foram realizados sob uma camada híbrida previamente formada, devido ao selamento dentinário imediato.

Com o auxílio de um pincel, o cimento resinoso dual (RelyX ARC, 3M ESPE, St. Paul, MN, USA) foi aplicado nos blocos de resina composta e espalhado homogeneamente. Logo após, os blocos foram assentados sobre os dentes preparados e os excessos de cimento removidos com pincel. Para concluir, os espécimes foram mantidos em posição por pressão digital e submetidos a fotoativação (Bluephase C5, Ivoclar Vivadent, Schaan, Liechtenstein) em todas as suas faces por 40s. Posteriormente, foi realizada a aplicação do gel hidrossolúvel (Power Block, BM4, Maringá, Brasil) e cada face foi fotoativada (Bluephase C5, Ivoclar Vivadent, Schaan, Liechtenstein) novamente por mais 20s. Concluída a cimentação, os corpos de prova foram mantidos em ambiente úmido por 7 dias, até a secção dos corpos de prova para o teste de microtração.

\section{Confecção dos Corpos de Prova}

0 conjunto dente/cimento/bloco foi fixado em uma máquina de secção (ISOMET 1000, Buehler Ltda; Lake Bluff, IL, EUA), onde os cortes foram efetuados com o auxílio de um disco diamantado dupla-face (4"X.012"X1/2", UKAM Industrial Superhard Tools; Valencia, CA, EUA) sob baixa velocidade $(125 \mathrm{rpm})$ e refrigeração constante. Os cortes foram primeiramente realizados no sentindo cérvico-incisal (eixo y) e, posteriormente, mésio-distal (eixo x), com a máquina ajustada para realizar cortes a cada 1,2 $\mathrm{mm}$. Como resultado obtiveram-se filetes de aproximadamente $1 \mathrm{~mm}^{2}$, mensurados por um paquímetro com leitura eletrônica (Lorben, São Paulo, SP, Brasil).

\section{Testes de Microtração}

Os corpos de prova foram sequencialmente fixados por suas extremidades ao dispositivo do teste de microtração, através de um adesivo a base de cianoacrilato (SuperBonder, Henkel Loctite Adesivos Ltda.; Itapevi, SP, Brasil) associado a um monômero de resina acrílica (Resinlay Speed Pattern, Pomerode, SC, Brasil). Cada filete foi posicionado de maneira que a linha de cimentação estivesse localizada perpendicularmente à força de microtração e de forma equidistante entre as garras dos dispositivos da máquina universal de ensaios (Instron, modelo 4444, Instron Corp, Canton, MA, EUA). A máquina foi configurada para realizar o teste a uma velocidade constante de $0,5 \mathrm{~mm} / \mathrm{min}$ e detectar o valor máximo de carga em Quilonewton $(\mathrm{kgN})$ necessária para a fratura dos corpos.

Por fim, os valores de carga registrados na ruptura dos corpos foram multiplicados por mil (x1000) para conversão de Quilonewton (kN) em Newton (N). Sequencialmente, os resultados em Newton (N) foram convertidos em MegaPascal (MPa), respeitando a equação: $\frac{\mathrm{N}}{\mathrm{mm}^{2}}=\mathrm{MPa}$. 


\section{ANÁLISE ESTATÍSTICA}

Para verificação dos resultados, os dados foram submetidos a análise estatística por meio de análise de variância (ANOVA). As análises foram realizadas usando SPSSStatics 26.0, e o valor de $\mathrm{p}$ foi definido como $5 \%$.

\section{RESULTADO}

Os valores médios da resistência de união à microtração ( $\mu$ TBS) para cada material restaurador estão descritos na Tabela 2. 0 teste ANOVA demonstrou diferença estatística entre os grupos avaliados $(\mathrm{p}=0,008)$. Observaram-se maiores valores de resistência de união (Figura 1) para o grupo SDIF (23,53 MPa), diferindo estatisticamente do Grupo $\mathrm{S}(\mathrm{p}=0,008)$. 0 grupo SDI apresentou valor médio de resistência de união $(19,25 \mathrm{MPa})$, não diferindo estatisticamente dos grupos $S(p=0,68)$ e SDIF $(p=0,07)$.

Tabela 2. Valores médios e desvio-padrão da resistência ao $\mu$ TSB frente aos tratamentos dos substratos dentinários

\begin{tabular}{cc}
\hline Grupos & $\boldsymbol{\mu T S B}$ (MPa) \\
\hline S & $17,65 \pm 6,5 \mathrm{~B}$ \\
SDI & $19,25 \pm 8,0 \mathrm{AB}$ \\
SDIF & $23,53 \pm 10,7 \mathrm{~A}$ \\
\hline
\end{tabular}

Letras MAIÚSCULAS iguais nas colunas indicam que não há diferença estatística entre os grupos (Teste ANOVA de um fator, post-hoc de Tukey $\mathrm{p}<0,05$ )

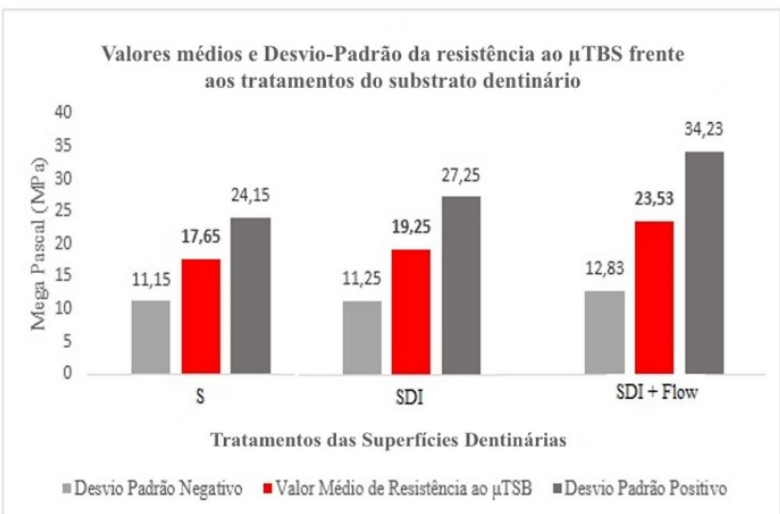

Figura 1. Valores médios e desvio-padrão da resistência ao $\mu \mathrm{TSB}$ frente aos tratamentos do substrato dentinário.

\section{DISCUSSÃO}

Alguns autores ${ }^{3,4}$ recomendam fortemente a realização do selamento dentinário imediato com o sistema adesivo de 3 passos específico de $4^{a}$ geração (Optibond ${ }^{\mathrm{TM}} \mathrm{FL}$; Kerr), pois a presença de carga em sua composição garantiria a formação de uma camada híbrida mais espessa, capaz de absorver a tensão de contração da resina ${ }^{12}$. Apesar de ser tratado como padrão ouro ${ }^{11}$ para execução da técnica do selamento dentinário imediato, a utilização desse sistema adesivo se torna dificultada por não ser facilmente encontrado no mercado nacional, e seu alto custo torna seu uso, muitas vezes, inviável.

Para efeito de comparação, na busca de resultados semelhantes com os adesivos autocondicionantes, que apresentam bons resultados ao serem utilizados na técnica do 
selamento dentinário imediato ${ }^{13,14}$, optou-se por um sistema adesivo de $5^{\text {a }}$ geração (Single Bond Universal, 3M ESPE, St Paul, MN, USA), ausente de cargas, porém, de fácil aquisição e, principalmente, por estar mais facilmente disponível para uso nas clínicas odontológicas. No presente estudo, o sistema adesivo de 5a geração (Single Bond Universal, 3M ESPE, St Paul, MN, USA) foi utilizado no modo autocondicionante, com intuito de aliar a redução de passos clínicos e os benefícios do selamento imediato ao da comodidade de usar um adesivo acessível para conduzir a prática clínica em quaisquer consultórios privados/públicos ou instituições de ensino.

Estudos avaliando a resistência de união de sistemas adesivos universais concluem que eles têm maior resistência de união do que outros tipos de sistemas adesivos convencionais, mostrando como hipótese para melhores resultados os componentes 10-MDP e ácido polialquenoico ${ }^{15-17}$, que são monômeros funcionais que aderem quimicamente ao cálcio da hidroxiapatita. 0 10-MDP encontra-se na composição da maioria dos adesivos universais. Especificamente, o sistema adesivo Single Bond Universal (3M ESPE, St Paul, MN, USA) apresenta, além do 10-MDP, um copolímero de ácido polialquenoico que também pode aderir quimicamente ao cálcio ${ }^{18}$. Isso conclui que tais componentes são o diferencial desse sistema adesivo universal.

0 grupo $\mathrm{S}$ simulou nesta pesquisa a abordagem tradicional de aplicação dos sistemas adesivos na prática clínica, através da hibridização e ativação do adesivo apenas momento da fixação final da restauração. Os resultados inferiores de adesão à dentina neste grupo eram esperados, uma vez que não é possível confeccionar e cimentar provisórios que selem totalmente a dentina. Estudos relatam que, para o selamento dentinário imediato, a dentina recém-exposta, livre de contaminantes, é considerada o substrato ideal. Isso porque a contaminação dentinária, que ocorre seja pelo contato com o cimento, a saliva e/ou componentes da dieta, pode ter como consequência a sensibilidade pós-operatória e respostas dolorosas ao tratamento ${ }^{1,2}$. Ainda, autores afirmam ${ }^{2,3}$ que reduções significativas na resistência adesiva à dentina podem ser desencadeadas quando uma contaminação com cimentos provisórios é simulada e comparada à dentina recém-exposta. 0 cimento residual temporário pode permanecer na superfície do dente mesmo após a limpeza mecânica, e alguns componentes do cimento penetram na superfície do dente, alterando características como ângulo de contato e permeabilidade dentinária. Dessa forma, a restauração definitiva geralmente não é aderida à dentina recém-preparada, mas sim à dentina contaminada, o que pode resultar em falha de hibridização e redução da resistência de união ${ }^{3}$.

Alguns estudos apresentam resultados contrários aos citados anteriormente, cuja resistência de união, quando a dentina é selada previamente à cimentação definitiva, é superior à resistência de união quando a dentina é selada no momento de sua exposição ${ }^{19,20 .}$

A diferença estatisticamente não significante entre o grupo S e o grupo SDI não foi prevista, pois esperavam-se maiores valores de união promovidos pelo selamento dentinário. Esse resultado pode ser explicado pela ausência de carga na composição do adesivo universal utilizado nesta pesquisa. Nos sistemas adesivos particulados, a camada híbrida formada é mais espessa21, sendo essa característica favorável à polimerização, já que, concluída a aplicação do adesivo, existe uma camada superficial inibida por oxigênio.

Os maiores valores registrados foram obtidos pelo grupo que recebeu o tratamento do selamento dentinário imediato e, sequencialmente, recobrimento da camada híbrida com resina flow (grupo SDIF). Os valores de resistência adesiva foram de 23,53 $\pm 10,7 \mathrm{MPa}$. Para este grupo, a qualidade da camada híbrida formada durante o selamento foi protegida por uma resina de baixa viscosidade e com partículas de carga em sua composição. Dessa forma, promoveu a proteção da dentina durante a fase de provisório e um aumento na resistência final de união entre o tecido dentário e o material restaurador após a cimentação adesiva. Ao aplicar uma camada adesiva hidrofóbica sobre uma superfície que foi tratada com um sistema adesivo de frasco único, a concentração de monômeros hidrofóbicos aumenta, consequentemente maiores são os índices de retenção e mais resistente se torna a interface adesiva à degradação ao longo do tempo ${ }^{22}$. 
Ainda assim, formulações específicas de cada adesivo, como a quantidade de monômeros hidrofóbicos e o solvente presente, podem afetar a qualidade da camada híbrida e a interação do adesivo com a resina flow ${ }^{23}$, o que pode explicar valores de resistência de união inferiores em alguns estudos.

Não foram registrados valores estatisticamente significantes entre os grupos SDI e SDIF, possivelmente porque a dentina tratada somente com o sistema adesivo teve a formação de uma camada híbrida de qualidade, porém recoberta por uma camada adesiva pouco resistente. Essa fragilidade na camada adesiva do grupo SDI pode ser consequência da formação de uma camada de adesivo muito delgada ou mesmo da redução da espessura da camada adesiva, seja pela cureta utilizada para remoção dos materiais provisórios aplicados sobre a dentina, pela profilaxia com escova robison e água ou pelo jateamento do espécime com óxido de alumínio. Assim, a hipótese avaliada neste estudo foi parcialmente aceita, uma vez que houve diferença estatisticamente significante entre a resistência de união à microtração da dentina dos grupos sem selamento dentinário e o selamento dentinário imediato com adesivo e recobrimento com resina flow.

Este trabalho teve o ensaio de microtração como método utilizado para avaliação da resistência de união adesiva. Nesse ensaio, a interface de união das amostras tem tamanho reduzido (cerca de $1 \mathrm{~mm}^{2}$ ), o que permite uma melhor distribuição de tensões durante o recebimento de carga. Consequentemente, há menos falhas coesivas na dentina do que as encontradas em modelos mais convencionais de testes. Ainda, o uso desse método geralmente resulta em valores de forças de adesão aparentes mais altas para ruptura dos espécimes do que as forças de adesão encontradas em amostras grandes. Ademais, permite a obtenção de vários corpos de prova a partir de um único dente ${ }^{24}$.

A utilização de dentes bovinos como possíveis substitutos de dentes humanos no teste de adesão à dentina pode ser justificada por estudos que não revelaram diferença estatisticamente significante entre os dentes humanos e bovinos quanto aos valores de resistência de união ao esmalte e à camada superficial da dentina ${ }^{25}$.

Entende-se que a extrapolação de resultados de estudos laboratoriais para a clínica deve ser feita sempre com cautela, uma vez que os estudos in vitro não conseguem reproduzir a real situação da cavidade oral. No entanto, o aumento da resistência de união encontrado para a dentina tratada com o selamento imediato associado à resina flow sugere efetividade da técnica, mesmo quando executada com um adesivo de $5^{\underline{a}}$ geração.

\section{CONCLUSÃO}

Foi concluído com este estudo que o selamento dentinário imediato realizado com o sistema adesivo universal e posterior recobrimento com resina flow deve ser realizado nas exposições dentinárias ocasionadas no preparo dental para restaurações indiretas.

\section{REFERÊNCIAS}

1. Magne P, Kim TH, Cascione D, Donovan TE. Immediate dentin sealing improves bond strength of indirect restorations. J Prosthet Dent. 2005 Dec;94(6):511-9. http://dx.doi.org/10.1016/j.prosdent.2005.10.010. PMid:16316797.

2. Bertschinger C, Paul SJ, Luthy H, Schärer P. Dual application of dentin bonding agents: effect on bond strength. Am J Dent. 1996 Jun;9(3):115-9. PMid:9002801.

3. Paul SJ, Schärer P. Effect of provisional cements on the bond strength of various adhesive bonding systems on dentine. J Oral Rehabil. 1997 Jan;24(1):8-14. http://dx.doi.org/10.1046/j.1365-

2842.1997.00484.x. PMid:9049913. 
4. Magne P. Immediate dentin sealing: a fundamental procedure for indirect bonded restorations. J Esthet Restor Dent. 2005;17(3):144-54, discussion 155. http://dx.doi.org/10.1111/j.17088240.2005.tb00103.x. PMid:15996383.

5. Dietschi D, Monasevic M, Krejci I, Davidson C. Marginal and internal adaptation of class II restorations after immediate or delayed composite placement. J Dent. 2002 Jul-Aug;30(5-6):259-69. http://dx.doi.org/10.1016/S0300-5712(02)00041-6. PMid:12450717.

6. Reis AF, Giannini M, Kavaguchi A, Soares CJ, Line SRP. Comparison of microtensile bond strength to enamel and dentin of human, bovine, and porcine teeth. J Adhes Dent. 2004;6(2):117-21. PMid:15293420.

7. Duarte S Jr, de Freitas CR, Saad JRC, Sadan A. The effect of immediate dentin sealing on the marginal adaptation and bond strengths of total-etch and self-etch adhesives. J Prosthet Dent. 2009 Jul;102(1):1-9. http://dx.doi.org/10.1016/S0022-3913(09)00073-0. PMid:19573687.

8. Albuquerque M, Pegoraro M, Mattei G, Reis A, Loguercio AD. Effect of double- application or the application of a hydrophobic layer for improved efficacy of one-step self-etch systems in enamel and dentin. Oper Dent. 2008 Sep-Oct;33(5):564-70. http://dx.doi.org/10.2341/07-145. PMid:18833863.

9. van den Breemer C, Özcan M, Cune MS, Ayres AA, Van Meerbeek B, Gresnigt M. Effect of immediate dentin sealing and surface conditioning on the microtensile bond strength of resin-based composite to dentin. Oper Dent. 2019 Nov-Dec;44(6):E289-98. http://dx.doi.org/10.2341/18-052-L. PMid:31084533.

10. De Goes MF, Giannini M, di Hipólito V, Carrilho MRO, Daronch M, Rueggeberg FA. Microtensile bond strength of adhesive systems to dentin with or without application of an intermediate flowable resin layer. Braz Dent J. 2008;19(1):51-6. http://dx.doi.org/10.1590/S0103-64402008000100009. PMid:18438560.

11. Jayasooriya PR, Pereira PNR, Nikaido T, Tagami J. Efficacy of a resin coating on bond strengths of resin cement to dentin. J Esthet Restor Dent. 2003;15(2):105-13, discussion 113. http://dx.doi.org/10.1111/j.1708-8240.2003.tb00325.x. PMid:12762474.

12. Van Meerbeek B, Peumans M, Poitevin A, Mine A, Van Ende A, Neves A, et al. Relationship between bond-strength tests and clinical outcomes. Dent Mater. 2010 Feb;26(2):e100-21. http://dx.doi.org/10.1016/j.dental.2009.11.148. PMid:20006379.

13. van den Breemer CR, Özcan M, Pols MR, Postema AR, Cune MS, Gresnigt MM. Adhesion of resin cement to dentin: effects of adhesive promoters, immediate dentin sealing strategies, and surface conditioning. Int J Esthet Dent. 2019;14(1):52-63. PMid:30714054.

14. Peumans M, Kanumilli P, De Munck J, Van Landuyt K, Lambrechts P, Van Meerbeek B. Clinical effectiveness of contemporary adhesives: a systematic review of current clinical trials. Dent Mater. 2005 Sep;21(9):864-81. http://dx.doi.org/10.1016/j.dental.2005.02.003. PMid:16009415.

15. Anchieta RB, Machado LS, Martini AP, dos Santos PH, Giannini M, Janal M, et al. Effect of long-term storage on nanomechanical and morphological properties of dentin-adhesive interfaces. Dent Mater. 2015 Feb;31(2):141-53. http://dx.doi.org/10.1016/j.dental.2014.11.010. PMid:25529501.

16. Jang J-H, Lee MG, Woo SU, Lee CO, Yi J-K, Kim D-S. Comparative study of the dentin bond strength of a new universal adhesive. Dent Mater J. 2016;35(4):606-12. http://dx.doi.org/10.4012/dmj.2015-422. PMid:27477226.

17. Schoenhals GP, Berft CL, Naufel FS, Schmitt VL, Chaves LP. Bond strength assessment of a universal adhesive system in etch-and-rinse and self-etch modes. Rev Odontol UNESP. 2019;48:e20190083. http://dx.doi.org/10.1590/1807-2577.08319.

18. Grégoire G, Sharrock P, Prigent Y. Performance of a universal adhesive on etched and non-etched surfaces: Do the results match the expectations? Mater Sci Eng C Mater Biol App. 2016 Sep;66:199-205. http://dx.doi.org/10.1016/j.msec.2016.04.022. PMid:27207055. 
19. Falkensammer F, Arnetzl GV, Wildburger A, Krall C, Freudenthaler J. Influence of different conditioning methods on immediate and delayed dentin sealing. J Prosthet Dent. 2014 Aug;112(2):204-10. http://dx.doi.org/10.1016/j.prosdent.2013.10.028. PMid:24787131.

20. da Silva CJR, Gonçalves ICS, Botelho MPJ, Guiraldo RD, Lopes MB, Gonini A Jr. Interactions between resin-based temporary materials and immediate dentin sealing. Appl Adhes Sci. 2016;4(3). http://dx.doi.org/10.1186/s40563-016-0061-9.

21. Davidson CL, Abdalla AI. Effect of oclusal load cycling on the marginal integrity of adhesive classe $\mathrm{V}$ restorations. Am J Dent. 1994 Apr;7(2):111-4. PMid:8054183.

22. Reis A, Albuquerque M, Pegoraro M, Mattei G, Bauer JRO, Grande RHM, et al. Can the durability of onestep self-etch adhesives be improved by double application or by an extra layer of hydrophobic resin? J Dent. 2008 May;36(5):309-15. http://dx.doi.org/10.1016/j.jdent.2008.01.018. PMid:18353520.

23. Japiassú Resende Montes MA, de Goes MF, Bernardi da Cunha MR, Borges Soares A. A morphological and tensile bond strength evaluation of an unfilled adhesive with low-viscosity composites and a filled adhesive in one and two coats. J Dent. 2001 Aug;29(6):435-41. http://dx.doi.org/10.1016/S03005712(01)00037-9. PMid:11520593.

24. Pashley DH, Carvalho RM, Sano H, Nakajima M, Yoshiyama M, Shono Y, et al. The microtensile bond test: a review. J Adhes Dent. 1999;1(4):299-309. PMid:11725659.

25. Nakamichi I, Iwaku M, Fusayama T. Bovine teeth as possible substitutes in the adhesion test. J Dent Res. 1983 Oct;62(10):1076-81. http://dx.doi.org/10.1177/00220345830620101501. PMid:6352757.

\section{CONFLITOS DE INTERESSE}

Os autores declaram não haver conflitos de interesse.

\section{*AUTOR PARA CORRESPONDÊNCIA}

Roberta Pinto Pereira, UFSC - Universidade Federal de Santa Catarina, Departamento de Odontologia, Rua Delfino Conti, 1240, Trindade, 88040-535 Florianópolis - SC, Brasil, e-mail: robertapkrohling@gmail.com

Recebido: Outubro 26, 2020

Aprovado: Novembro 18, 2020 